\title{
Multicanonical parallel tempering
}

\author{
Roland Faller, Qiliang Yan, and Juan J. de Pablo \\ Department of Chemical Engineering, University of Wisconsin, Madison, Wisconsin 53706
}

(Received 29 May 2001; accepted 10 January 2002)

\begin{abstract}
We present a novel implementation of the parallel tempering Monte Carlo method in a multicanonical ensemble. Multicanonical weights are derived by a self-consistent iterative process using a Boltzmann inversion of global energy histograms. This procedure gives rise to a much broader overlap of thermodynamic-property histograms; fewer replicas are necessary in parallel tempering simulations, and the acceptance of trial swap moves can be made arbitrarily high. We demonstrate the usefulness of the method in the context of a grand-multicanonical ensemble, where we use multicanonical simulations in energy space with the addition of an unmodified chemical potential term in particle-number space. Several possible implementations are discussed, and the best choice is presented in the context of the liquid-gas phase transition of the Lennard-Jones fluid. A substantial decrease in the necessary number of replicas can be achieved through the proposed method, thereby providing a higher efficiency and the possibility of parallelization. (C) 2002 American Institute of Physics. [DOI: 10.1063/1.1456504]
\end{abstract}

\section{INTRODUCTION}

Advanced Monte Carlo simulation techniques can facilitate the study of complex systems considerably. Two classes of methods that have proven to be particularly useful are parallel tempering techniques ${ }^{1-5}$ (sometimes also called multiple Markov chains), and multicanonical techniques. ${ }^{6-8}$ A recent review of these methods can be found in the literature. $^{9}$

In parallel tempering, several independent replicas of a system are simulated simultaneously. Each replica (or simulation box) can experience different thermodynamic-state conditions (e.g., temperature, pressure, or chemical potential). Neighboring systems (in the sense that their state points are not too distant from each other) are allowed to interchange configurations from time to time, subject to specific acceptance criteria. These so-called "swap" moves can improve sampling of configuration space considerably, particularly in systems having rugged energy landscapes. Replicas of a system which are close to a glassy state, for example, may exchange their way "up" in, say, temperature, to states where energy barriers are easier to overcome; they can subsequently come back to low temperatures to yield an uncorrelated configuration.

More specifically, $t$ independent replicas of the same system are simulated under different thermodynamic conditions, $C_{1}, C_{2}, \ldots, C_{t}$, where the $C_{i}$ denote combinations of intensive variables (e.g., temperature and chemical potential) which differ from replica to replica. Conventional Monte Carlo trial moves are conducted in each replica $i$ to sample configuration space. In addition, trial swap moves involving two replicas $i$ and $j$ are also attempted; in these trial moves, entire conformations are interchanged. The acceptance criteria for a trial swap can be derived from the product of the elementary moves which are used to construct it. We use subscripts $m$ and $n$ to denote the configurations pertaining to two distinct replicas, or simulation boxes; $i$ and $j$ are used to denote their respective thermodynamic conditions. In the particular case of a grand-canonical ensemble, the probability of accepting an individual trial move from configuration $m$ to $n$ at reduced inverse temperature $\beta_{i}=1 / k_{B} T_{i}$ and chemical potential $\mu_{i}$ is given by

$$
p_{i}=\min \left\{1, \exp \left[-\beta_{i}\left(E_{n}-E_{m}\right)+\beta_{i} \mu_{i}\left(N_{n}-N_{m}\right)\right]\right\},
$$

where $N_{n}$ denotes the number of particles in replica $n$. That of accepting an individual trial move from configuration $n$ to $m$ at temperature $T_{j}$ and chemical potential $\mu_{j}$ is given by

$$
p_{j}=\min \left\{1, \exp \left[-\beta_{j}\left(E_{m}-E_{n}\right)+\beta_{j} \mu_{j}\left(N_{m}-N_{n}\right)\right]\right\} .
$$

A swap move can be viewed as a "double-move," for which the acceptance probability is the product of $p_{i}$ and $p_{j}$ :

$$
\begin{aligned}
p_{i j}= & \min \left\{1, \exp \left[-\beta_{i}\left(E_{n}-E_{m}\right)-\beta_{j}\left(E_{m}-E_{n}\right)\right.\right. \\
& \left.\left.+\beta_{i} \mu_{i}\left(N_{n}-N_{m}\right)+\beta_{j} \mu_{j}\left(N_{m}-N_{n}\right)\right]\right\} \\
= & \min \left\{1, \exp \left[-\left(\beta_{i}-\beta_{j}\right)\left(E_{n}-E_{m}\right)\right.\right. \\
& \left.\left.+\left(\beta_{i} \mu_{i}-\beta_{j} \mu_{j}\right)\left(N_{n}-N_{m}\right)\right]\right\} .
\end{aligned}
$$

From Eq. (3) it can be seen that swap trial moves are only accepted if some degree of overlap exists between the probability distribution functions (or histograms) corresponding to neighboring state points (or replicas). One shortcoming of parallel tempering is that the number of replicas required for an effective simulation increases with the size of the simulated system. This is a result of the central limit theorem, which shows that the width of thermodynamicproperty histograms scales as $\sqrt{N}^{-1}$, thereby decreasing the extent of overlap between histograms corresponding to neighboring replicas.

Parallel tempering simulations improve sampling by shuttling configurations from regions of low temperature or high chemical potential to regions of high temperature or low chemical potential, where a system can relax more easily. They have the added feature that each of the replicas gener- 
ates useful information (e.g., thermodynamic quantities, structure) about the system of interest. Multicanonical simulations follow an entirely different strategy to overcome high-energy barriers between neighboring, local free-energy minima: The acceptance criteria for the transition between two states are manipulated in such a way as to artificially lower such barriers. The conventional energy distribution for a canonical ensemble involves two contributions: the density of states $\Omega(E)$ and a Boltzmann, exponential energy weight of the form $\exp [-\beta E]$. On the one hand, the density of states increases rapidly with energy and system size and, on the other hand, the exponential energy term leads to a suppression of high-energy states when the energy exceeds the thermal energy significantly. The product of the density of states and the Boltzmann weight therefore results in a Gaussianlike energy distribution. In multicanonical simulations, the conventional Boltzmann weight is replaced by a different, non-Boltzmann weight, $w_{\mathrm{NB}}$, which is conceived in such a way as to result in a flat energy distribution. A flat distribution would be desirable for two reasons: from a statisticalmechanics point of view, realizing a perfectly flat energy distribution is equivalent to calculating the density of states of the system (or its microcanonical-ensemble partition function); the logarithm of this quantity is the entropy. From a more technical point of view, realizing a flat energy distribution ensures that all states are sampled with comparable frequency, thereby improving statistics.

For concreteness, the following discussion is restricted to one-dimensional distributions depending only on energy; the extension to multidimensional cases is straightforward. The probability $p(E)$ of finding the system of interest in a given energy state can be expressed in the form

$$
p(E)=\Omega(E) w(E) .
$$

Equation (4) is valid regardless of the weights $w(E)$; different weights characterize different ensembles. For a canonical, NVT-ensemble, $w_{\mathrm{NVT}}(E)=\exp (-\beta E)$. In multicanonical simulations a final set of weights is calculated in such a way as to make $p(E)$ flat, i.e., independent of $E$. A perfectly flat distribution could be generated if the following weights were employed

$$
w(E)=\frac{1}{\Omega(E)}=\exp \left(-S(E) / k_{B}\right),
$$

where $S(E)$ is the entropy as a function of energy. In view of the form of Eq. (5), simulation techniques in which different energy states are sampled with uniform probability are sometimes referred to as entropic sampling methods.

To calculate properties using a uniform-energy, or multicanonical sampling technique, energy histograms $H_{\mathrm{mc}}(E)$ are generated during the course of the simulation. These histograms provide estimates of the probability of finding a configuration having energy $E$ in a multicanonical ensemble; they can subsequently be "reweighted" in order to generate results in one of the more conventional ensembles. The socalled multicanonical weights, which dictate the sampling, are denoted by $w_{\mathrm{mc}}(E)$. Their construction is discussed later in this work. Taking the internal energy as an example, we have

$$
\langle E\rangle_{\mathrm{can}}=\sum_{E} \frac{\exp (-\beta E)}{w_{\mathrm{mc}}(E)} H_{\mathrm{mc}}(E) E,
$$

where the brackets denote a canonical ensemble average.

The two methods, parallel tempering and multicanonical simulations, have several attributes of their own. It is therefore of interest to explore the possible advantages of a combined method, in which parallel tempering would be used to have independent random walkers in different parts of the energy landscape, and multicanonical Monte Carlo would be employed to reduce the number of necessary replicas. This work investigates such a combination. Ideas similar in spirit were pursued by Sugita et al. ${ }^{10}$ and Calvo and Doye. ${ }^{11}$ Sugita's work, however, only considered single-molecule simulations. It is unclear whether the Sugita et al. approach would be of use in a many-body system. The work of Calvo and Doye proposes a scheme that differs from ours in that it involves exchanges between one multicanonical trajectory and multiple tempering replicas. Furthermore, that work is also limited to single molecules or small atomic clusters, and therefore does not address many of the issues that arise in many-body, condensed phases.

\section{MULTICANONICAL PARALLEL TEMPERING}

In this work, we have chosen to implement a multicanonical sampling scheme through a so-called umbrella potential $\xi(E)$, which is added to the energy in the grand canonical ensemble. Our multicanonical weights are of the form

$$
w_{\mathrm{mc}}(E, N)=\exp [-\beta(E+\xi(E))+\beta \mu N] .
$$

The factor $\exp (-\beta \xi(E))$ changes the distribution from Boltzmann to one possible type of multicanonical. In order to satisfy Eq. (5), the ideal umbrella potential $\xi(E)$ should be of the form

$$
\xi(E)=-E+T S(E) .
$$

This would lead to purely entropic sampling in that all energy states would be visited with equal frequency, according to $1 / \Omega(E)$.

Unfortunately, the entropy of a system to be simulated is not known a priori; the calculation of $\xi$ must therefore be carried out through a self-consistent, iterative process. A series of simulations are conducted; the umbrella potential is adjusted in such a way as to render the energy landscape corresponding to each simulation successively flatter, i.e., the "weights" of formerly poorly visited states are augmented, and those of more heavily visited states are reduced.

Our starting point is a grand-canonical, multidimensional parallel tempering simulation where we set $\xi^{(0)}(E)$ $=0$ over the entire energy range. Upper indices refer to iteration numbers. We use one single, global $\xi(E)$ for all replicas. Otherwise, an uncontrolled bias would lead to incorrect estimates of the histograms and ultimately incorrect results. After a few thousand simulation cycles, we analyze the global energy histogram derived from all the replicas

$$
H(E)=\sum_{i} H_{i}(E),
$$


where $H_{i}(E)$ is the energy histogram collected in replica $i$. This histogram is now "Boltzmann inverted" [Eq. (10)], i.e., its logarithm is multiplied by $k_{B} T$ to obtain an estimate of the corresponding weight. The current value of $\xi^{(n)}(E)$ is then updated according to

$$
\xi^{(n+1)}(E)=\xi^{(n)}(E)+\beta_{\max }^{-1} \ln H(E)-\beta_{\max }^{-1} \overline{\ln H(E)} .
$$

The third term in Eq. (10) is a constant, and it corresponds to the average over all of the $\ln H(E)$; it drops out of any acceptance criteria. Its sole purpose is computational efficiency. It allows the umbrella potentials to increase as well as decrease between iterations (its omission leads to more iterations).

Different replicas are simulated at different temperatures; we must therefore choose the particular temperature at which to perform the operations involved in Eq. (10). Note that if all the replicas were at the same temperature, the Boltzmann inversion would yield the free-energy difference between iterations $n$ and $n+1$. In our case, however, it is a corrective procedure for the weights employed in the simulation, which were designed to produce a flatter distribution. We find that inversion at the minimum temperature (which corresponds to the maximum $\beta$, denoted by $\beta_{\max }$ ) provides an optimum choice. At first glance, inverting every histogram at the temperature at which it was generated and adding up the resulting umbrellas would appear to be the best choice. This implementation was successful in the sense that it leads to flatter distributions, but it doubled the number of iterations required to achieve the same accuracy as that attained by inversion at the lowest temperature. This is due to the fact that the error of the histograms is highest in the flanks. Adding up two umbrellas with large errors but correct temperature is less efficient than adding up the histograms of different temperatures and inverting the global histogram. Tests conducted by inverting at intermediate temperatures always overestimated the low-temperature barriers, leading to insufficient sampling in formerly highly frequented areas. In some cases the histograms were even shifted to completely new areas; Fig. 1 illustrates that effect. In that case, a low-temperature replica $(T=0.79)$ was shifted into a region of little interest by inverting at a temperature in the middle of the range covered by all the replicas $(T=1)$. The region of little interest corresponds in this case to energies, which are only relevant at much lower temperatures; the energies that we set out to sample were no longer visited. Alternatively, an even lower temperature (lower than the minimum temperature of the simulation) could be used for inversion; in that case, however, the efficiency of the algorithm deteriorates considerably, as not even the lowest-temperature histogram becomes sufficiently flat.

In the first iteration, the Boltzmann weight $-\beta_{i} E$ $+\beta_{i} \mu_{i} N$ is exchanged by $-\beta_{i}\left(E+\xi^{(1)}(E)\right)+\beta_{i} \mu_{i} N$. The change of weights between two successive iterations $n$ and $n+1$ can therefore be expressed as
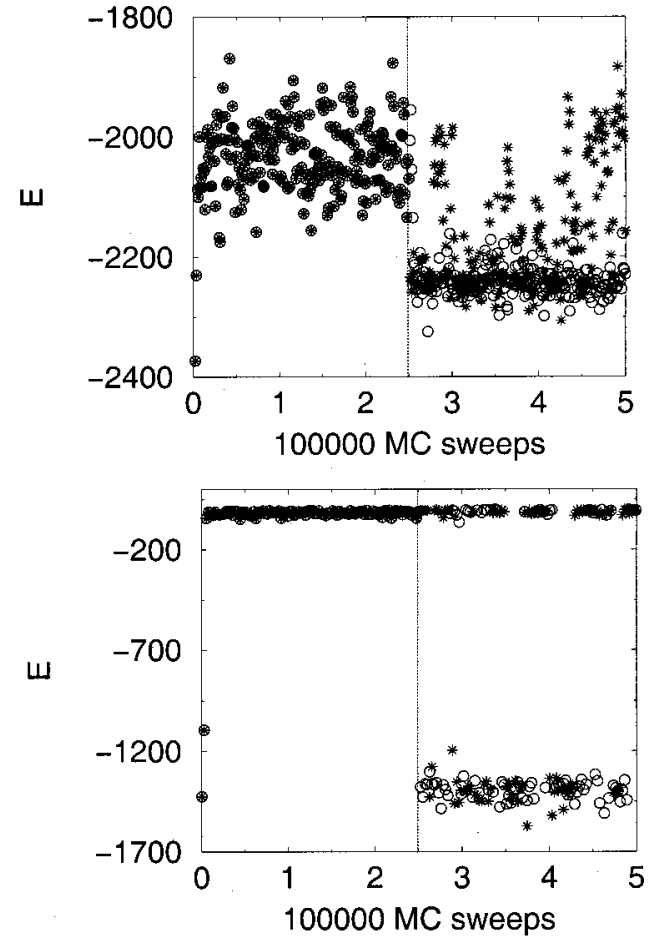

FIG. 1. Upper: Energy of the lowest temperature replica $(T=0.79)$ as a function of Monte Carlo sweeps. Circles: Inversion temperature $T^{\text {invert }}=1$, stars: $T^{\text {invert }}=T_{\min }=0.79$. After 250000 steps (dotted line) the first umbrella is incorporated in the simulation. The high inversion temperature umbrella leads to a complete suppression of sampling of the relevant energies. Lower: Same as the upper figure, but for the replica at $T=1.03$. For clarity we only show data every 25 cycles.

$$
\begin{aligned}
w_{\mathrm{mc}}^{(n+1)}(E) & =\frac{w_{\mathrm{mc}}^{(n)}(E)}{\Omega^{\prime}(E)} \\
& =w_{\mathrm{mc}}^{(n)}(E) \exp \left\{-\beta\left[\xi^{(n+1)}(E)-\xi^{(n)}(E)\right]\right\} \\
& =w^{(0)}(E) \exp \left\{-\beta \xi^{(n+1)}(E)\right\},
\end{aligned}
$$

where $w^{(0)}$ denotes the original, grand canonical ensemble weight [i.e., $\left.w^{(0)}=\exp (-\beta E+\beta \mu N)\right]$. For the swap moves in the Metropolis criteria of Eq. (3), the energy $E$ is interchanged with the umbrella-corrected energy, $E+\xi(E)$.

\section{RESULTS FOR THE LENNARD-JONES FLUID}

The Lennard-Jones fluid provides a standard test for the study of new methods, partly because highly accurate data are available for comparison. ${ }^{5,12,13}$ In this work, we apply the multicanonical parallel tempering method described above to determine its vapor-liquid phase behavior. The interaction potential is given by

$$
\begin{aligned}
& \Gamma(r)=4 \epsilon\left[\left(\frac{\sigma}{r}\right)^{12}-\left(\frac{\sigma}{r}\right)^{6}\right], r<r_{c}=2.5 \sigma, \\
& \Gamma(r)=0, \quad r \geqslant r_{c} .
\end{aligned}
$$

All data are reported in standard dimensionless units (distances are measured in $\sigma$, energies and temperature in $\epsilon$ ). The box length is $L=8 \sigma$; the grand canonical ensemble is used as the parent ensemble for the simulation. The multicanonical umbrellas are one-dimensional and are only functions of 

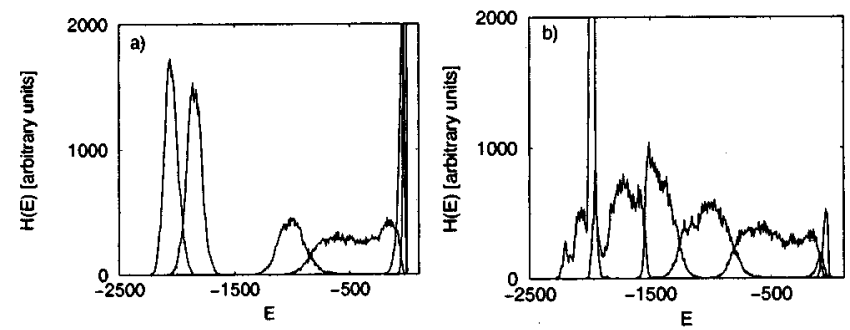

FIG. 2. Energy histograms with and without application of the multicanonical weights used to calculate the Lennard-Jones phase diagram. Part a shows the histograms of the potential energy per replica without multicanonical simulation, part b with application of the multicanonical weights, which were produced in four iterations containing $250000 \mathrm{MC}$ cycles each.

the energy $\xi=\xi(E)$. A two-dimensional implementation of the umbrella $[\xi=\xi(E, N)]$ was also attempted, but this deteriorated the overall performance of the algorithm and is not presented here. After several days of computer time, our twodimensional implementation of the algorithm had not converged. When two-dimensional umbrellas are employed, the initial runs must be much longer to fill the histograms and reduce their statistical uncertainty. Furthermore, addressing a two-dimensional table (as opposed to a simple vector) hinders computational performance. A possible solution to this problem could perhaps be found through the twodimensional generalization ${ }^{14}$ of the recently proposed Density of States Monte Carlo method. ${ }^{15}$ That method, however, is in its early stages of development and it is unclear whether it will work for large systems.

The preliminary runs to produce the umbrella were performed with between $50 * 10^{3}$ and $250 * 10^{3}$ MC cycles for every $\xi^{(i)}(E)$. A swap between neighboring replicas was attempted every 100 Monte Carlo cycles. The last $60 \%$ of these cycles were used to create the energy histograms for inversion in the following step. Equilibration was determined by the decay of the autocorrelation function of the energy, and by the fact that the histograms did not change significantly over time. The histograms generated using only $20 * 10^{3}, 50 * 10^{3}$, or $250 * 10^{3}$ Monte Carlo were essentially undistinguishable. The correlation time was a few hundred MC cycles, which corresponds to a few successful swaps. These findings suggest that in the particular case of a Lennard-Jones fluid (at liquid-like densities), $20 * 10^{3}$ Monte Carlo cycles are sufficient to equilibrate the system in the new umbrella for subsequent histogram collection.

The multicanonical weights are initially calculated as outlined above, until a desired swap rate between neighboring replicas is achieved; a good acceptance probability is about $5 \%$ to $10 \%$. For six replicas, the sought-after overlap between neighboring histograms was attained after four iterations (see Fig. 2). Note that, by design, the energy histograms corresponding to our original choice of state points (Table I) did not overlap with each other. The temperatures and chemical potentials were chosen as a subset from earlier parallel tempering simulations with 18 replicas. ${ }^{5}$ Conventional parallel-tempering swap moves would not work for that choice. After applying the multicanonical weights, overlap between all replica histograms was achieved, thereby leading to a highly effective parallel tempering simulation.
TABLE I. Thermodynamic conditions for a grand canonical ensemble tempering simulation using six replicas. These conditions are taken as starting points for the multicanonical optimization. These thermodynamic conditions are taken from the literature (Ref. 5).

\begin{tabular}{ccc}
\hline \hline$i$ & $\mathrm{~T}_{i}$ & $\mu_{i}$ \\
\hline 1 & 0.79 & -4.76 \\
2 & 0.86 & -4.23 \\
3 & 1.03 & -3.35 \\
4 & 1.11 & -3.02 \\
5 & 1.20 & -2.74 \\
6 & 0.94 & -3.98 \\
\hline \hline
\end{tabular}

We performed two independent simulations of $10^{6}$ steps; only data produced over the last three quarters of the simulation were used for analysis. Note that in cases where we used a different inversion temperature to produce the umbrellas, or in cases were every histogram was inverted at its temperature (see above), we did not find any significant changes in the results; the production of the histograms, however, was considerably slower. As a word of caution, we point out that it is important that the umbrella potentials be based on well-equilibrated histograms. This can cut down the number of iteration steps significantly, especially in the initial stages of the process where the weights change drastically between successive iterations.

In order to determine the optimal distribution of state points, we also performed simulations using four and eight state points (or replicas) for multicanonical parallel tempering simulations. For reference, we also conducted a conventional canonical parallel tempering simulation with 12 and 18 state points. Again, the state points were subsets taken from Ref. 5. A one replica, purely multicanonical simulation was attempted for reference; that simulation was not successful in covering the entire region of interest (with a single replica) and the initial runs did not converge. The number of iterations needed for the calculation of the umbrellas is shown in Table II, along with the time required for production runs. We find that with four replicas we needed an unreasonably high number of iterations, and with eight replicas we could not cut down on the number of iterations required with respect to six replicas. For the size of our simulated system it appears that six replicas provide an optimal choice. It is important to emphasize, however, that the optimum is

TABLE II. Number of iterations used for calculation of the umbrellas $N_{u}$, number of iterations times number of replicas $\left(N \cdot N_{u}\right)$, and overall CPU time $T_{\mathrm{CPU}}$ (in hours) required to simulate the relevant system on a simple personal computer ( $800 \mathrm{MHz}$ AMD processor). For the case of one replica, we were unable to produce an individual umbrella capable of covering the entire coexistence region.

\begin{tabular}{cccc}
\hline \hline \# replicas & \# iterations & \# of iterations $\times \#$ replicas & CPU time \\
$N$ & $N_{u}$ & $N \cdot N_{u}$ & $T_{\mathrm{CPU}} / \mathrm{h}$ \\
\hline 1 & $>80$ & $>80$ & $>80$ \\
4 & 11 & 44 & 76 \\
6 & 4 & 24 & 72 \\
8 & 5 & 40 & 80 \\
12 & $\ldots$ & $\ldots$ & 120 \\
18 & $\ldots$ & $\cdots$ & 180 \\
\hline \hline
\end{tabular}



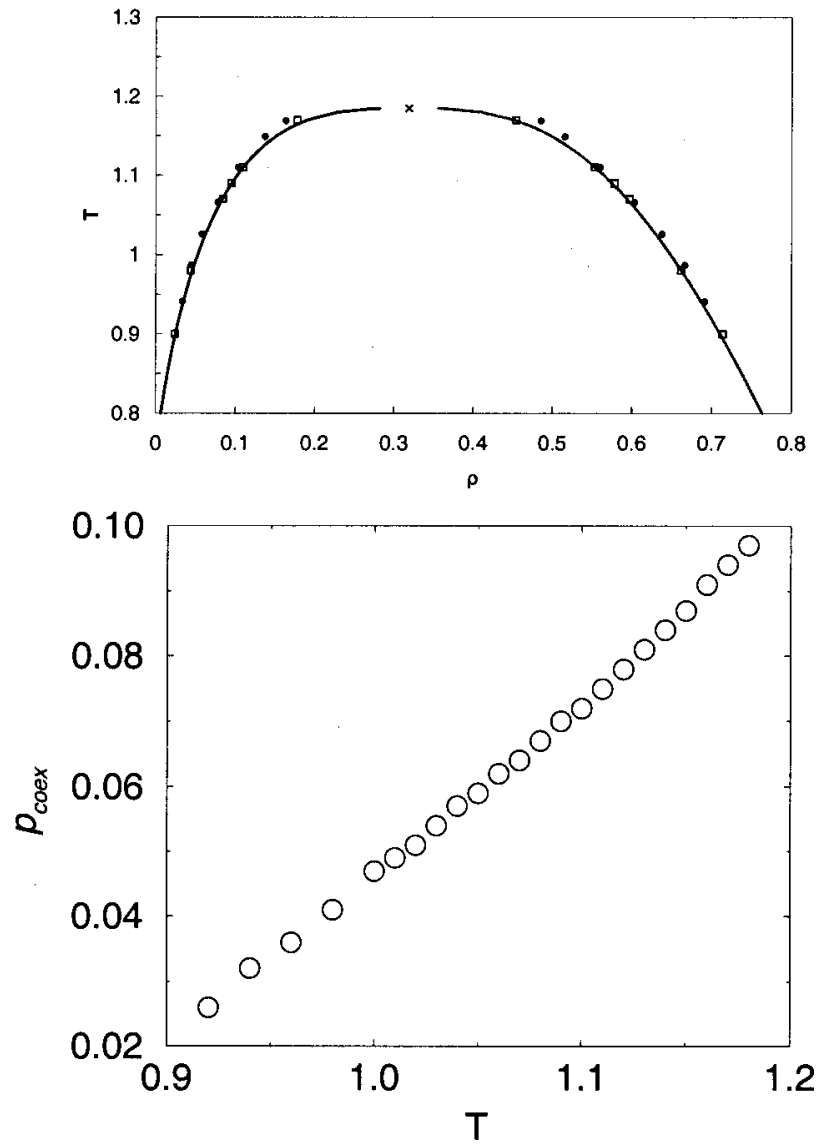

FIG. 3. Upper: Calculated Phase Diagram for the Lennard-Jones fluid. The open squares show results of this work. The circles show literature data (Ref. 13). The solid line is an Ising fit to the data. Lower: Coexistence (vapor) pressure as a function of temperature for the same system.

likely to vary significantly depending on the particular details of a given system. Of course, the time required for the final production run scales with the number of replicas; from this point of view, the proposed algorithm is three times more efficient than the original 18-replica multidimensional parallel-tempering simulation of the same system. ${ }^{5}$

The new method permits coverage of the entire coexistence region using only six simulation replicas. One additional, important advantage over a traditional paralleltempering simulation resides in the smaller size of the overall system, which reduces drastically memory requirements. For systems like glasses or polymers, a large number of replicas would be necessary for parallel tempering simulations; such calculations would require extraordinary computational resources, as the amount of memory scales linearly with the number of replicas. For a large polymeric system, an individual replica containing several tens of thousands of interaction sites is already at the limits of a standard personal computer or workstation. With the combined parallel-tempering-multicanonical approach proposed here, such systems can be simulated using a smaller number of replicas, thereby reducing the computational requirements considerably (both memory and production run time).

Figure 3 shows the phase diagram and the coexistence pressure as a function of temperature calculated using the above-mentioned six replicas. We use multihistogram re- weighting to calculate the other state points, ${ }^{16,17}$ as there is good overlap in the histograms the accuracy of the reweighting procedure increases. The phase diagram is in good agreement with literature data. ${ }^{5,13}$ The estimated critical temperature and density are $T_{c}=1.18 \pm 0.01, \rho_{c}=0.32 \pm 0.02$. These were obtained by an Ising fit to the reweighted data. We did not apply a finite-size scaling correction.

\section{CONCLUSIONS}

We have presented an implementation of multicanonical sampling in the framework of parallel-tempering simulations. This combination of methods decreases the number of necessary replicas and increases the efficiency of the simulation. The validity of the new method has been demonstrated in the context of a simple Lennard-Jones fluid, for which high-accuracy literature data for the coexistence curve are reproduced.

As pointed out in the multicanonical-ensemble literature, we note that it is important to exercise care in the selection of multicanonical weights. However, when proper precautions are followed, the result is an effective methodology. Even for simple systems, such as the Lennard-Jones fluid, the memory and time required to produce results over a wide range of conditions can be decreased by a factor of three without an overhead to the overall calculation. We expect the proposed formalism to be particularly well-suited for simulation of systems which, by their very nature, require large sizes (e.g., polymers). An additional, important advantage of the proposed method is that it provides a remarkably simple way of parallelizing an umbrella-sampling simulation; to the best of our knowledge, the implementation presented here constitutes the first version of an umbrella sampling simulation that is parallelized.

\section{ACKNOWLEDGMENTS}

One of the authors (R.F.) thanks Markus Deserno for fruitful discussions. This work was supported by the Division of Chemical Sciences, Office of Basic Energy Sciences, Office of Sciences, U.S. Department of Energy. Acknowledgment is also made to the Donors of the Petroleum Research Fund, administered by the American Chemical Society. R.F. is supported by the Emmy-Noether program of the German Research Foundation (DFG).

${ }^{1}$ E. Marinari and G. Parisi, Europhys. Lett. 19, 451 (1992).

${ }^{2}$ M. C. Tesi, E. J. J. van Rensburg, E. Orlandini, and S. G. Whittington, J. Stat. Phys. 82, 155 (1996).

${ }^{3}$ U. H. E. Hansmann, Chem. Phys. Lett. 281, 140 (1997).

${ }^{4}$ M. G. Wu and M. W. Deem, Mol. Phys. 97, 559 (1999).

${ }^{5}$ Q. Yan and J. J. de Pablo, J. Chem. Phys. 111, 9509 (1999).

${ }^{6}$ G. M. Torrie and J. P. Valleau, J. Comput. Phys. 23, 187 (1977).

${ }^{7}$ B. A. Berg and T. Neuhaus, Phys. Rev. Lett. 68, 9 (1992).

${ }^{8}$ B. A. Berg, Fields Inst. Commun. 26, 1 (2000).

${ }^{9}$ Y. Iba, Int. J. Mod. Phys. C 12, 623 (2001).

${ }^{10}$ Y. Sugita, A. Kitao, and Y. Okamoto, J. Chem. Phys. 113, 6042 (2000).

${ }^{11}$ F. Calvo and J. P. K. Doye, Phys. Rev. E 63, 010902 (2000).

${ }^{12}$ B. Smit, J. Chem. Phys. 96, 8639 (1992).

${ }^{13}$ N. B. Wilding, Phys. Rev. E 52, 602 (1995).

${ }^{14}$ Q. Yan, R. Faller, and J. J. de Pablo, J. Chem. Phys. (in press).

${ }^{15}$ F. Wang and D. P. Landau, Phys. Rev. Lett. 86, 2050 (2001).

${ }^{16}$ A. M. Ferrenberg and R. H. Swendsen, Phys. Rev. Lett. 61, 2635 (1988).

${ }^{17}$ A. M. Ferrenberg and R. H. Swendsen, Phys. Rev. Lett. 63, 1195 (1989). 